\title{
Lack of support for an association between CLEC4M homozygosity and protection against SARS coronavirus infection
}

\section{To the Editor:}

Chan et al. ${ }^{1}$ reported that individuals homozygous for a tandem repeat polymorphism (VNTR) in exon 4 of CLEC4M were protected against SARS coronavirus infection (odds ratio of 0.7 ), whereas heterozygotes were more susceptible to infection. This repeat region encodes 윽 the extracellular neck domain of the L-SIGN ('liver/lymph node-specific ICAM-3 grabbing nonintegrin') molecule, which is responsible for oligomerization into a functional tetramer. Functional studies by Chan et al. suggested that the protective effect was due to formation of a homotetramer of L-SIGN, with apparently higher affinity for viral ligands, in homozygous subjects. However, the authors also indicated that a similar protective effect was observed in cells that expressed L-SIGN with only two repeats (see Supplementary Fig. 6 in Chan et al.). This finding is not consistent with the hypothesis that formation of homotetrameric
L-SIGN accounts for protection against trans infection, because L-SIGN with two repeats cannot form stable tetramers ${ }^{2,3}$, and monomeric receptors show much lower affinity and avidity for viral ligands ${ }^{3}$. Furthermore, the presence of a variety of alternatively spliced CLEC4M mRNAs, including isoforms with partial deletion in the neck region, suggests that the correlation between genotype and function may not be a simple one $e^{4}$.

As about half of the Chinese population consists of heterozygotes, the results of Chan et al., if confirmed, bear important public health implications for SARS susceptibility. We tried to replicate these findings with another collection of 177 individuals with SARS. All affected individuals had a laboratory-confirmed diagnosis of SARS infection by either PCR tests for SARS coronavirus or serology. We studied three independent control samples of Hong Kong Chinese: (i) anonymous archival cord blood samples
( $n=463$ ), to determine population genotype frequencies; (ii) healthy elderly individuals aged $>70$ years $(n=163)$, to determine if age had any effect on genotype frequencies and (iii) a further sample collected from local university students ( $n=248)$. A fourth population sample collected in Beijing (in northern China) was used to determine if there was any subpopulation structure (that is, variation of allele frequencies across different parts of China) for this polymorphism. We purified genomic DNA from whole-blood samples and performed PCR to genotype the VNTR in exon 4 of CLEC4M using the same protocol as described previously ${ }^{1}$. We confirmed genotype calls by duplicated assays.

Genotype frequencies and homozygote proportions are shown in Table 1. The genotype distributions and homozygote proportions of the three groups of controls were not different from those of individuals with SARS ( $P=$ 0.72). Genotype frequencies of all samples,

Table 1 Genotype distributions and homozygote proportions of CLEC4M neck region tandem repeat polymorphism in individuals with SARS and controls

\begin{tabular}{|c|c|c|c|c|c|c|c|c|c|c|}
\hline \multirow{2}{*}{$\begin{array}{l}\text { CLEC4M tandem repeat } \\
\text { genotypes } \\
5 / 5\end{array}$} & \multicolumn{2}{|c|}{ Archival cord blood samples } & \multicolumn{2}{|c|}{ Healthy elderly controls } & \multicolumn{2}{|c|}{ University students } & \multicolumn{2}{|c|}{ Individuals with SARS } & \multicolumn{2}{|c|}{ Beijing controls ${ }^{a}$} \\
\hline & 17 & $3.7 \%$ & 1 & $0.6 \%$ & 3 & $1.2 \%$ & 4 & $2.3 \%$ & 5 & $2.5 \%$ \\
\hline $5 / 9$ & 20 & $4.3 \%$ & 3 & $1.8 \%$ & 3 & $1.2 \%$ & 8 & $4.5 \%$ & 8 & $4.0 \%$ \\
\hline $6 / 5$ & 8 & $1.7 \%$ & 1 & $0.6 \%$ & 5 & $2.0 \%$ & 6 & $3.4 \%$ & 1 & $0.5 \%$ \\
\hline $6 / 9$ & 5 & $1.1 \%$ & 4 & $2.5 \%$ & 6 & $2.4 \%$ & 4 & $2.3 \%$ & 1 & $0.5 \%$ \\
\hline $7 / 4$ & 1 & $0.2 \%$ & 0 & $0.0 \%$ & 0 & $0.0 \%$ & 0 & $0.0 \%$ & 0 & $0.0 \%$ \\
\hline $7 / 5$ & 94 & $20.3 \%$ & 33 & $20.2 \%$ & 52 & $21.0 \%$ & 30 & $16.9 \%$ & 39 & $19.4 \%$ \\
\hline $7 / 6$ & 46 & $9.9 \%$ & 15 & $9.2 \%$ & 13 & $5.2 \%$ & 11 & $6.2 \%$ & 10 & $5.0 \%$ \\
\hline $7 / 7$ & 189 & $40.8 \%$ & 68 & $41.7 \%$ & 99 & $39.9 \%$ & 76 & $42.9 \%$ & 102 & $50.7 \%$ \\
\hline $7 / 9$ & 75 & $16.2 \%$ & 30 & $18.4 \%$ & 61 & $24.6 \%$ & 35 & $19.8 \%$ & 30 & $14.9 \%$ \\
\hline $9 / 8$ & 1 & $0.2 \%$ & 1 & $0.6 \%$ & 0 & $0.0 \%$ & 0 & $0.0 \%$ & 0 & $0.0 \%$ \\
\hline $9 / 9$ & 7 & $1.5 \%$ & 7 & $4.3 \%$ & 6 & $2.4 \%$ & 3 & $1.7 \%$ & 5 & $2.5 \%$ \\
\hline Total & 463 & & 163 & & 248 & & 177 & & 201 & \\
\hline Homozygotes & 213 & $46.0 \%$ & 76 & $46.6 \%$ & 108 & $43.5 \%$ & $83^{b}$ & $46.9 \%$ & 112 & $55.7 \%$ \\
\hline Heterozygotes & 250 & $54.0 \%$ & 87 & $53.4 \%$ & 140 & $56.5 \%$ & 94 & $53.1 \%$ & 89 & $44.3 \%$ \\
\hline
\end{tabular}


except the group of university students $(P=$ 0.028), were in Hardy-Weinberg equilibrium (by a Markov chain method in GENEPOP). Furthermore, we compared the genotypes among individuals with SARS with different prognoses. If L-SIGN homozygosity is a protective factor against infection, it may also be associated with better prognosis after acquiring the infection. Therefore, we also examined whether homozygotes had a better prognosis by classifying individuals with SARS who had an uneventful recovery versus those who had severe disease and were admitted to the intensive care unit for mechanical ventilation support (an approach similar to that reported previously $\left.{ }^{5}\right)$. However, we did not detect any significant association ( $P=0.9$, Supplementary Table 1 online).

Sample size is the main limitation of both studies. However, these two samples already represent the few 'large' collections of individuals with SARS available for genetic study. To estimate the size of an overall effect, we performed a meta-analysis of the two data sets together by the Mantel-Haenszel test using control groups in Hardy-Weinberg equilibrium (two groups of controls in this study and random controls from Chan et al.; total $n=1,468$; 462 affected individuals and 1,006 controls). The combined odds ratio was not significant (combined $\mathrm{OR}=0.84 ; 95 \%$ confidence interval: $0.66-1.06, P=0.14)$.

The difference in the results between the two studies was basically accounted for by a difference in the homozygote proportions in the controls $(45.4 \%$ in this study versus $55.0 \%$ in Chan et al.), while the homozygote proportions among individuals with SARS are almost identical (46.9\% here versus $46.3 \%$ in Chan et al.). The reason for the discrepancy in the homozygote proportions in the 'control' groups is not clear. However, a subpopulation difference in allelic and genotypic frequencies exists between northern and southern Chinese. The seven-repeat allele was more prevalent in the Beijing sample (0.7 in Beijing versus 0.64 in Hong Kong; $P=0.05$ ), which also largely accounted for the higher proportion of homozygotes (55.7\% in Beijing versus $46.0 \%$ in Hong Kong; $P=0.02$ ). Unrecognized subpopulation structure may confound genetic association studies. Results in the study by Chan et al. suggested that this confounding factor might be present. There were three groups of controls, including two groups of hospital controls (health care workers who worked in SARS wards and affected individuals attending various outpatient clinics) and a group of blood donor controls. Interestingly, genotype distributions from both groups of hospitalbased controls deviated significantly $(P<$ $0.0001)$ or marginally $(P=0.05)$ from HardyWeinberg equilibrium.

In addition, other yet-unknown mechanisms (such as alternative splicing of the neck region, which could interfere with formation of homotetramers among homozygotes) may account for the discrepancy between the two studies. Replication is an important approach to verify any significant genetic association findings ${ }^{6,7}$, and additional association studies are required to establish the putative protective effect of L-SIGN homozygosity against SARS or other infections.

Nelson Leung-Sang Tang ${ }^{1,2}$, Paul Kay-Sheung $\mathrm{Chan}^{3}$, David Shu-Cheong Hui ${ }^{4,5}, \mathrm{Ka}-\mathrm{Fai} \mathrm{To}^{6}$, Weimin Zhang ${ }^{7}$, Francis K L Chan ${ }^{4}$, Joseph JaoYiu Sung ${ }^{4,5}$ \& Yuk Ming Dennis Lo ${ }^{1,2}$

${ }^{1}$ Department of Chemical Pathology, ${ }^{2} \mathrm{Li} \mathrm{Ka}$ Shing Institute of Health Sciences, ${ }^{3}$ Department of Microbiology, ${ }^{4}$ Department of Medicine and Therapeutics, ${ }^{5}$ Stanley Ho Centre for Emerging Infectious Diseases, and ${ }^{6}$ Department of Anatomical and Cellular Pathology, Faculty of Medicine, The Chinese University of Hong Kong, Hong Kong, People's Republic of China. ${ }^{7}$ Peking Union Medical College Hospital, Beijing, People's Republic of China.

e-mail:nelsontang@cuhk.edu.hk

Note: Supplementary information is available on the Nature Genetics website.

\section{COMPETING INTERESTS STATEMENT}

The authors declare no competing financial interests.

1. Chan, V.S. et al. Nat. Genet. 38, 38-46 (2006).

2. Feinberg, H. et al. J. Biol. Chem. 280, 1327-1335 (2005).

3. Snyder, G.A. et al. J. Virol. 79, 4589-4598 (2005).

4. Liu, H. et al. Eur. J. Hum. Genet. 13, 707-715 (2005).

5. Tang, N.L. et al. Clin. Chem. 51, 2333-2340 (2005).

6. Ott, J. Neurology 63, 955-958 (2004).

7. Anonymous. Nat. Genet. 22, 1-2 (1999).

\section{Lack of support for an association between CLEC4M homozygosity and protection against SARS coronavirus infection}

To the Editor:

In the January 2006 issue, Chan et al. ${ }^{1}$ reported a significant association between severe acute respiratory syndrome (SARS) and a variable number of tandem repeats (VNTR) polymorphism in exon 4 of CLEC4M in a collection of individuals from Hong Kong. CLEC4M encodes L-SIGN ('liver/lymph node-specific ICAM-3 grabbing nonintegrin'), which serves as a receptor for many viruses, including SARS coronavirus $(\mathrm{CoV})^{2}$. Individuals homozygous for CLEC4M tandem repeats were reported to be less susceptible to SARS CoV infection. The authors also showed that cells homozygous for CLEC4M repeats had a higher binding capacity for SARS CoV, higher proteasome-dependent viral degradation and a lower capacity for trans infection. Thus, both genetic and functional studies suggested that homozygosity for CLEC4M was associated with protection against SARS CoV infection.

It is important to bear in mind that association studies require replication in independent populations ${ }^{3}$. We therefore attempted to replicate the findings of Chan et al. by genotyping the VNTR polymorphism in three additional collections of case-control samples from northern China: (i) the 'Beijing community population', consisting of 339 individuals with SARS and 227 random controls recruited from the community'; (ii) the 'Beijing health care worker (HCW) population', consisting of 42 health care workers infected with SARS during the course of hospital duty and 40 health care workers who had worked in SARS wards but remained free of disease and were confirmed to be seronegative for SARS 5 and (iii) the 'Tianjin population', consisting of 60 individuals with SARS and 129 disease-free controls (including 85 random controls and 44 health care workers) ${ }^{6}$. The three collections of case-control samples and their ascertainment criteria have been described in detail previously (Supplementary Methods online $)^{4-6}$. All groups except the individuals with SARS from the Beijing community were in Hardy-Weinberg equilibrium. We found no significant differences in allele, genotype and homozygote or heterozygote frequencies between affected individuals and controls in the three populations (Table 1 and Supplementary Table 1 online). Early reports 